\title{
Transabdominal Resection
}

National Cancer Institute

\section{Source}

National Cancer Institute. Transabdominal Resection. NCI Thesaurus. Code C153341.

Surgery comprising either abdominoperineal resection, low anterior resection, or coloanal anastomosis with total mesorectal excision. 